\title{
High power, short duration ablation: at least for the right pulmonary vein carina, maybe not so fast
}

\author{
Gustavo Guandalini ${ }^{1}$ and Francis Marchlinski ${ }^{2}$ \\ ${ }^{1}$ University of Pennsylvania \\ ${ }^{2}$ Hospital of the University of Pennsylvania
}

March 3, 2021

High power, short duration ablation: at least for the right pulmonary vein carina, maybe not
so fast

Gustavo S. Guandalini, MD ${ }^{\text {a }}$ and Francis E. Marchlinski, MD ${ }^{a}$

a Section of Cardiac Electrophysiology, Hospital of the University of Pennsylvania, Philadelphia, PA

Corresponding author: Dr. Francis E. Marchlinski, Division of Cardiovascular Medicine, Electrophysiology Section, Hospital of the University of Pennsylvania, 3400 Spruce Street, 9 Founders Pavilion, Philadelphia, Pennsylvania 19104. E-mail: Francis.Marchlinski@pennmedicine.upenn.edu.

The authors have no conflicts to disclose.

Word count: 1,170 .

This research was supported by the Richard T and Angela Clark Innovation Fund in Cardiovascular Medicine.

\section{Editorial}

Catheter ablation for atrial fibrillation (AF) has undergone successive technical advances that incrementally improved its performance. Such evolution reached a stage of maturity that made it the most common procedure performed by electrophysiologists. In the past few years, radiofrequency (RF) ablation using high-power, short duration (HPSD) settings (50W for 5 to 10 seconds) has been advocated as a safe and effective strategy to achieve point-by-point pulmonary vein isolation. $(1,2)$ But before universally adopting such a strategy, electrophysiologists should be familiarized with the biophysics of RF ablation and understand what makes HPSD ablation unique in this setting.

In contrast to conventional ablation, which relies on passive heat conduction into deeper tissue for transmural lesion formation (thus requiring longer RF application at lower power), HPSD relies on resistive heat from the catheter-tissue interface for effective ablation. (3) This produces much less conductive heat leading to wider, shallower lesion formation and much less reversible injury (figure 1 ). In AF ablation, in which injury to deep tissue beyond the thin left atrial wall is of major concern, the arguments in favor of HPSD can thus potentially extend beyond faster lesion formation and shorter procedural time. For those who remain skeptical, however, the question yet to be answered is: 'Can HPSD improve efficacy of AF ablation while maintaining equal or higher safety compared to more standard energy delivery techniques?'.

In this issue of the Journal of Cardiovascular Electrophysiology, Hansom et al. address this question by systematically reporting outcomes using HPSD ablation - defined as 50W for 6 to 8 seconds posteriorly and 8 to 10 seconds elsewhere - to low-power, long duration (LPLD) ablation - defined as up to $25 \mathrm{~W}$ with force-time integral (FTI) of at least 300g.s posteriorly and up to $35 \mathrm{~W}$ with FTI of at least 400g.s elsewhere. 
This well-designed, well-executed historical control study was based on a change in strategy at their center in 2017, allowing a comparison between 107 consecutive patients who underwent AF ablation with LPLD settings with 107 consecutive for whom HPSD settings were used. Although not a randomized controlled trial, patients in both groups had similar baseline characteristics, including similar proportion of paroxysmal and persistent AF (respectively, $63 \%$ and $37 \%$ in the HPSD group versus $56 \%$ and $44 \%$ in the LPLD group).

In terms of safety, their findings supported the notion that HPSD ablation is at least as safe as LPLD ablation. Specifically, there were no steam pops and no atrioesophageal fistulas in their series; however, the only case of phrenic nerve palsy happened in the HPSD group. Although not powered to detect statistically significant differences with such low event rates, this observation is a reminder that HPSD ablation could still lead to unintended complications, highlighting the importance of phrenic nerve pacemapping prior to right pulmonary vein isolation regardless of the power settings. Their overall low complication rate is in agreement with prior studies that also indicate no increase in complication rates when HPSD is compared to LPLD ablation. $(1,4-6)$

In regard to efficacy, Hansom et al. showed that HPSD achieved results comparable to LPLD, but with shorter ablation and procedure times. Acute procedural success (defined as pulmonary vein isolation with bidirectional block) was achieved in all patients, with an average procedural time of 229 minutes and 25.8 minutes of ablation in the HPSD group compared to 309 and 64.8 minutes, respectively, in the LPLD group ( $\mathrm{p}<0.005$ for both parameters). Freedom from atrial arrhythmias after 1 year was similar between groups: $79 \%$ in HPSD and $73 \%$ in LPLD ( $\mathrm{p}<0.004$ for non-inferiority). The same was true when AF ablation outcome was further stratified by type. Again, these findings were consistent with previous reports that suggest at least equivalent short- and long-term outcomes when HPSD is employed instead of LPLD. $(4,7,8)$

The most powerful message from this paper, however, is revealed after a closer look into how HPSD ablation performed in different segments of the pulmonary veins. While acute pulmonary vein isolation was accomplished in all patients, additional right pulmonary vein carinal lesions were required in almost twice as many patients in the HPSD group compared to LPLD $(32 \%$ versus $17 \%, \mathrm{p}=0.011)$. Furthermore, among patients who underwent repeat procedure due to recurrent atrial arrhythmias, late reconnection was concentrated on right pulmonary vein carina segments in HPSD patients, while a more even distribution of reconnected segments was seen in LPLD patients. This higher proportion of right carinal reconnection (respectively $47 \%$ versus $21 \%, \mathrm{p}=0.035$ ) was observed in spite of no significant difference in overall number of reconnected segments or reconnected veins per patient.

This intriguing observation requires an explanation, and the authors offer some insight as to why this might be the case. An increased tissue thickness at these sites explains worse performance of HPSD ablation, as thicker muscle would require more conductive heating for deeper lesion formation. (7) However, linear ablations in thick tissue often performed concomitantly during AF ablation (such as cavotricuspid-isthmus ablation, left atrial roof and anteroseptal mitral annulus lines) would also perform poorly with HPSD, which is not supported by current evidence but admittedly not studied in detail. $(9,10)$ The current authors also previously demonstrated that the right pulmonary vein carina is more susceptible to catheter instability, (11) largely explained by the more complex local anatomy as well as more challenging catheter manipulation given close proximity to transseptal access site.

Therefore, caution should be taken when excellent catheter stability cannot be achieved. In those instances, longer lesion duration at lower power might be more effective than much faster energy applications, with proportionally more time in true contact with tissue and better current delivery. (12) If HPSD eventually becomes the preferred approach for AF ablation, care should also be taken when ablating the posterior wall immediately adjacent to the esophagus or when ablating near the phrenic nerve, given the potential for less reversible collateral injury with HPSD. In these regions, high power lesion duration of 2 or 3 seconds longer may be enough to cause unintended irreversible tissue injury to adjacent structures and the time limits of 'short duration' still need to be better defined. In the future, perhaps a hybrid approach alternating HPSD and LPLD settings in different sites of the left atrium would potentially become the optimal approach. Hansom and colleagues are to be congratulated for a job well done in both confirming the value and documenting 
a remaining challenge when using HPSD for pulmonary vein isolation.

\section{References}

1. Bhaskaran A, Chik W, Pouliopoulos J, Nalliah C, Qian P, Barry T, et al. Five seconds of 50-60 W radio frequency atrial ablations were transmural and safe: an in vitro mechanistic assessment and force-controlled in vivo validation. Europace. 2017 May 1;19(5):874-880.

2. Qiu J, Wang Y, Wang DW, Hu M, Chen G. Update on high-power short-duration ablation for pulmonary vein isolation. J Cardiovasc Electrophysiol. 2020 Sep;31(9):2499-2508.

3. Leshem E, Zilberman I, Tschabrunn CM, Barkagan M, Contreras-Valdes FM, Govari A, et al. HighPower and Short-Duration Ablation for Pulmonary Vein Isolation. JACC: Clinical Electrophysiology. 2018 Apr;4(4):467-479.

4. Barkagan M, Contreras-Valdes FM, Leshem E, Buxton AE, Nakagawa H, Anter E. High-power and short-duration ablation for pulmonary vein isolation: Safety, efficacy, and long-term durability. J Cardiovasc Electrophysiol. 2018 Jun 20;29(9):1287-1296.

5. Winkle RA, Mohanty S, Patrawala RA, Mead RH, Kong MH, Engel G, et al. Low complication rates using high power $(45-50 \mathrm{~W})$ for short duration for atrial fibrillation ablations. Heart Rhythm. 2019;16(2):165-169.

6. Kewcharoen J, Techorueangwiwat C, Kanitsoraphan C, Leesutipornchai T, Akoum N, Bunch TJ, et al. High-power short duration and low-power long duration in atrial fibrillation ablation: A meta-analysis. J Cardiovasc Electrophysiol. 2021 Jan;32(1):71-82.

7. Yavin HD, Leshem E, Shapira-Daniels A, Sroubek J, Barkagan M, Haffajee CI, et al. Impact of High-Power Short-Duration Radiofrequency Ablation on Long-Term Lesion Durability for Atrial Fibrillation Ablation. JACC Clin Electrophysiol. 2020;6(8):973-985.

8. Ravi V, Poudyal A, Abid Q-U-A, Larsen T, Krishnan K, Sharma PS, et al. High-power short duration vs. conventional radiofrequency ablation of atrial fibrillation: a systematic review and meta-analysis. Europace. 2021 Feb 1;

9. Golian M, Ramirez FD, Alqarawi W, Hansom SP, Nery PB, Redpath CJ, et al. High-power short-duration radiofrequency ablation of typical atrial flutter. Heart Rhythm O2. 2020 Dec;1(5):317-323.

10. Zanchi S, Chen S, Bordignon S, Bianchini L, Tohoku S, Bologna F, et al. Ablation Index guided highpower $(50 \mathrm{~W})$ short-duration for left atrial anterior and roof line ablation: feasibility, procedural data and lesion analysis (AI High-Power Linear Ablation). J Cardiovasc Electrophysiol. 2021 Feb 26;

11. Dhillon G, Ahsan S, Honarbakhsh S, Lim W, Baca M, Graham A, et al. A multicentered evaluation of ablation at higher power guided by ablation index: Establishing ablation targets for pulmonary vein isolation. J Cardiovasc Electrophysiol. 2019 Jan 6;30(3):357-365.

12. Bourier F, Sommer P. The shorter, the better?: short duration ablation, catheter stability, and lesion durability. JACC Clin Electrophysiol. 2020;6(8):986-988.

Figures 


\section{Low-power, long duration}

At $4 \sec$ (initial ablation phase)

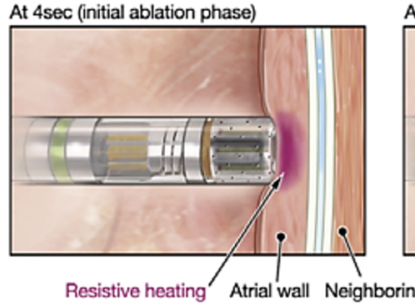

structures

structures
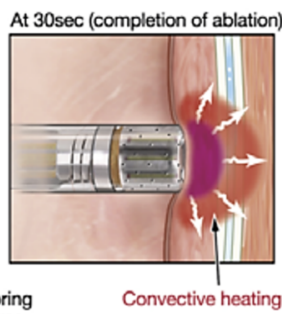

Convective heating

High-power, short duration

At $4 \mathrm{sec}$ (completion of ablation)

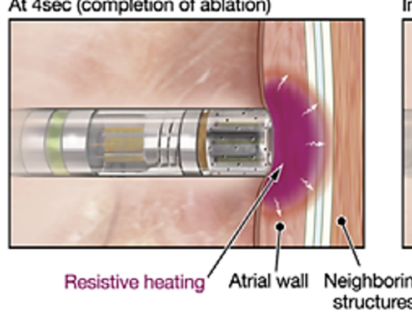

Immediately post-ablation

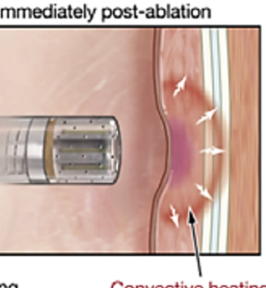

Convective heating
Acute lesion

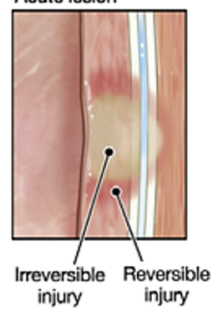

injury

Acute lesion

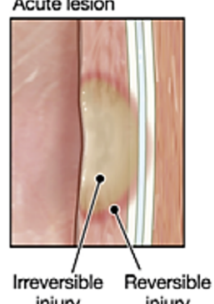

Chronic lesion

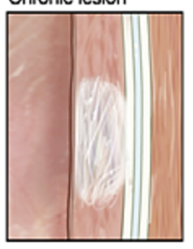

Figure 1. Conceptual difference in lesion formation between low-power, long duration ablation (LPLD, top row) and high-power, short duration ablation (HPSD, bottom row). In LPLD lesions, there is significantly more conductive heating leading to a wider area of reversible injury and deeper lesion formation. In contrast, HPSD relies mostly on resistive heating from the catheter-tissue interface, with much less reversible injury and potentially wider, shallower lesion formation. (Modified with permission from Barkagan M, ContrerasValdes FM, Leshem E, Buxton AE, Nakagawa H, Anter E. High-power and short-duration ablation for pulmonary vein isolation: Safety, efficacy, and long-term durability. J Cardiovasc Electrophysiol. 2018; 29(9):1287-1296.)

\section{Hosted file}

Figure.pptx available at https://authorea.com/users/399366/articles/511925-high-power-shortduration-ablation-at-least-for-the-right-pulmonary-vein-carina-maybe-not-so-fast 